\title{
A Holographic Dark Energy Model from Ricci Scalar Curvature
}

\author{
Changjun Gao, Fengquan Wu, and Xuelei Chen \\ The National Astronomical Observatories, Chinese Academy of Sciences, Beijing, 100012, China \\ You-Gen Shen \\ Shanghai Astronomical Observatory, Chinese Academy of Sciences, Shanghai 200030, China and \\ Joint Institute for Galaxy and Cosmology of SHAO and USTC, Shanghai 200030, China
}

(Dated: November 2008)

\begin{abstract}
Motivated by the holographic principle, it has been suggested that the dark energy density may be inversely proportional to the area of the event horizon of the Universe. However, such a model would have a causality problem. In this paper, we propose to replace the future event horizon area with the inverse of the Ricci scalar curvature. We show that this model does not only avoid the causality problem and is phenomenologically viable, but also naturally solves the coincidence problem of dark energy. Our analysis of the evolution of density perturbations show that the matter power spectra and CMB temperature anisotropy is only slightly affected by such modification.
\end{abstract}

PACS numbers: $98.80 . \mathrm{Cq}, 98.65 . \mathrm{Dx}$

\section{INTRODUCTION}

Ever since the discovery of the dark energy [1, 2], cosmologists are confronted with two fundamental problems: (1) the fine tuning problem and (2) the coincidence problem (see e.g. [3]). The fine tuning problem is the following: the simplest form of dark energy is the cosmological constant introduced by Einstein. However, the vacuum energy in quantum field theory has exactly the same property, and the estimated size of the vacuum energy is $\rho \simeq \rho_{p}$ where $\rho_{p} \sim m_{p}^{4}$ is the Plank density. It is greater than the observed value $\rho \simeq 10^{-123} \rho_{p}$ by some 123 orders of magnitude, so extreme fine tuning of the vacuum energy is required. The coincidence problem is the following: the density of the dark energy and matter evolves differently as the Universe expands, yet they are comparable today, this is an incredibly great coincidence if there is not some internal connection between the two.

An important advance in the studies of black hole theory and string theory is the suggestion of the so called holographic principle, which may provide some clue for solving these problems. It is realized that in quantum gravity, the entropy of a system scales not with its volume, but with its surface area $L^{2}[4]$. To see how this could help solve the cosmological constant problems, we note that in the Einstein equation, $G_{\mu \nu}=8 \pi G T_{\mu \nu}+$ $\Lambda g_{\mu \nu}$, the cosmological constant $\Lambda$ is the inverse of some length squared, $[\Lambda] \sim l^{-2}$, and to be consistent with observations, $l$ must be of the same order as the present cosmological scale. It is then proposed [5] that an unknown vacuum energy could be present, and according to the holographic principle its density is proportional to the Hubble scale $l_{H} \sim H^{-1}$. In this model the fine tuning problem is solved as the scale of dark energy is determined not by Planck length but by cosmological scale, and the coincidence problem is also all alleviated. Unfortunately, the effective equation of state for such vacuum energy is zero and the Universe is decelerating. Alterna- tively, the particle horizon size $l_{P H}=a \int_{0}^{t} d t / a$ could be used as the length scale [6]. However, as S. Hsu 7] and M. Li [8] pointed out, the equation of state for this dark energy model is greater than $-1 / 3$, so it still could not explain the observed acceleration of the Universe. In view of this, M. Li [8] proposed that the future event horizon of the Universe to be used as the characteristic length l. This holographic dark energy model and its interacting versions are successful in fitting the current observations [9].

However, the underlying origin of the holographic dark energy is still unknown. Furthermore, the model also has some serious conceptual problems. As R. Cai [10] pointed out, an obvious drawback concerning causality appears in this proposal. Event horizon is a global concept of space-time. However, the density of dark energy is a local quantity. Why should a local quantity be determined by a global one? Also puzzling is that the present value of dark energy is determined by the future evolution of the Universe, thus posing a challenge to the concept of casuality. Furthermore, for a spatially flat FriedmannRobertson-Walker Universe, it is well-known that the future event horizon exists if and if the Universe is accelerating. So in order to interpret the cosmic acceleration, the holographic dark energy model itself has presumed the acceleration.

Inspired by the holographic dark energy models, in this paper we propose to consider another possibility: the length $l$ is giving by the average radius of Ricci scalar curvature, $R^{-1 / 2}$, so that we have the dark energy $\rho_{X} \propto$ $R$. In the following we shall call this model the Ricci dark energy model, and investigate its phenomenological properties. We find that this model works fairly well in fitting the observational data, and it could also help us to understand the coincidence problem. Moreover, in this model the presence of event horizon is not presumed, so the causality problem is avoided.

In the next section we describe the model and its cosmic expansion history. Section III is devoted to the 
study of structure formation and CMB anisotropy in this model. In Section IV, we summarize our results, and discuss various problems, including how it solves the finetuning and coincidence problem, while avoids the problem of causality. We also discuss possible physical mechanism for such a dark energy. We also show that one could construct a K-essence model to mimic its behavior in the Appendix. Throughout the paper we adopt the Planck units, i.e. $c=\hbar=G=1$.

\section{THE MODEL}

The metric of the Friedmann-Robertson-Walker Universe is given by

$$
d s^{2}=-d t^{2}+a(t)^{2}\left(\frac{d r^{2}}{1-k r^{2}}+r^{2} d \theta^{2}+r^{2} \sin ^{2} \theta d \phi^{2}\right)
$$

where $k=1,0,-1$ for closed, flat and open geometries respectively. In this we have adopted the convention of $a_{0}=1$, where the subscript 0 denotes the value at present time (zero redshift). The Friedman equation is

$$
H^{2}=\frac{8 \pi}{3} \sum_{i} \rho_{i}-\frac{k}{a^{2}}
$$

where $H \equiv \dot{a} / a$ is the Hubble parameter, dot denotes the derivative with respect to the cosmic time $t$, and summation runs over the non-relativistic matter, radiation and other components. The Ricci scalar curvature is given by

$$
R=-6\left(\dot{H}+2 H^{2}+\frac{k}{a^{2}}\right)
$$

We consider a dark energy component, which arises from unknown physics and is proportional to the inverse of squared Ricci scalar curvature radius as prescribed by the holographic principle,

$$
\rho_{X}=\frac{3 \alpha}{8 \pi}\left(\dot{H}+2 H^{2}+\frac{k}{a^{2}}\right)=-\frac{\alpha}{16 \pi} R,
$$

where $\alpha$ is a constant to be determined. The factor $\frac{3}{8 \pi}$ before $\alpha$ is for convenience in the following calculations. The corresponding energy-momentum tensor can be written as:

$$
T_{\mu \nu}=\left(\rho_{X}+p_{X}\right) U_{\mu} U_{\nu}+p_{X} g_{\mu \nu}
$$

where $U_{\mu}$ is the 4-velocity of the co-moving observer, and $p_{X}$ is the pressure of dark energy. Setting $x=\ln a$, we can rewrite the Friedmann equation as follows

$$
\begin{aligned}
H^{2}= & \frac{8 \pi}{3}\left[(\alpha-1) \frac{3 k}{8 \pi} e^{-2 x}+\rho_{m} e^{-3 x}+\rho_{r} e^{-4 x}\right] \\
& +\alpha\left(\frac{1}{2} \frac{d H^{2}}{d x}+2 H^{2}\right),
\end{aligned}
$$

where $\rho_{m}$ and $\rho_{r}$ term are the contributions of nonrelativistic matter and radiation, respectively. We introduce the scaled Hubble expansion rate $h \equiv H / H_{0}$, then the above Friedman equation becomes

$$
\begin{aligned}
h^{2}= & (\alpha-1) \Omega_{k 0} e^{-2 x}+\Omega_{m 0} e^{-3 x}+\Omega_{r 0} e^{-4 x}+ \\
& \alpha\left(\frac{1}{2} \frac{d h^{2}}{d x}+2 h^{2}\right)
\end{aligned}
$$

where $\Omega_{k 0}, \Omega_{m 0}$ and $\Omega_{r 0}$ are the relative density of the curvature, non-relativistic matter and radiation in the present Universe, and the dark energy relative density is denoted by $\Omega_{X}$, with $\Omega_{k 0}+\Omega_{m 0}+\Omega_{r 0}+\Omega_{X 0}=1$. Solving Eq.(7), we obtain

$$
\begin{aligned}
h^{2}= & -\Omega_{k 0} e^{-2 x}+\Omega_{m 0} e^{-3 x}+\Omega_{r 0} e^{-4 x}+ \\
& \frac{\alpha}{2-\alpha} \Omega_{m 0} e^{-3 x}+f_{0} e^{-\left(4-\frac{2}{\alpha}\right) x},
\end{aligned}
$$

where $f_{0}$ is an integration constant. On the right hand side of Eq.(8), the last two terms come from the dark energy,

$$
\rho_{X}=\frac{\alpha}{2-\alpha} \Omega_{m 0} e^{-3 x}+f_{0} e^{-\left(4-\frac{2}{\alpha}\right) x} .
$$

Thus the Ricci dark energy has one part which evolves like non-relativistic matter $\left(\sim e^{-3 x}\right)$, and another part which is slowly increasing with decreasing redshift.

We assume that energy is conserved in such model, substituting the expression of $\rho_{X}$ into the conservation equation of energy,

$$
p_{X}=-\rho_{X}-\frac{1}{3} \frac{d \rho_{X}}{d x}
$$

we obtain the pressure of dark energy

$$
p_{X}=-\left(\frac{2}{3 \alpha}-\frac{1}{3}\right) f_{0} e^{-\left(4-\frac{2}{\alpha}\right) x} .
$$

There are two constants $\alpha$ and $f_{0}$ to be determined in the expressions of $\rho_{X}$ and $p_{X}$. If the density $\Omega_{X}$ and equation of state $w_{0} \Omega_{X}$ of the dark energy is known, the value of $\alpha$ and $f_{0}$ can be determined using Eq. (9) and Eq. (11):

$$
\frac{\Omega_{m 0} \alpha}{2-\alpha}+f_{0}=\Omega_{X 0}, \quad-\left(\frac{2}{3 \alpha}-\frac{1}{3}\right) f_{0}=w_{0} \Omega_{X 0} .
$$

We then obtain

$$
f_{0}=\frac{3 w_{0} \Omega_{X 0}^{2}}{3 w_{0} \Omega_{X 0}-\Omega_{m 0}}, \quad \alpha=\frac{2 \Omega_{X 0}}{\Omega_{m 0}+\Omega_{X 0}-3 w_{0} \Omega_{X 0}} .
$$

As an example, we consider the cosmology model with the following values of parameters: $\Omega_{k 0}=0, \Omega_{m 0}=$ $0.27, \Omega_{r 0}=8.1 \cdot 10^{-5}, \Omega_{X 0}=0.73, w_{0}=-1$, which are consistent with current observations [11], then we find $f_{0} \simeq 0.65$ and $\alpha \simeq 0.46$. We plot the evolution of the equation of state $w \equiv p_{X} / \rho_{X}$ for this model in Fig. 1. 


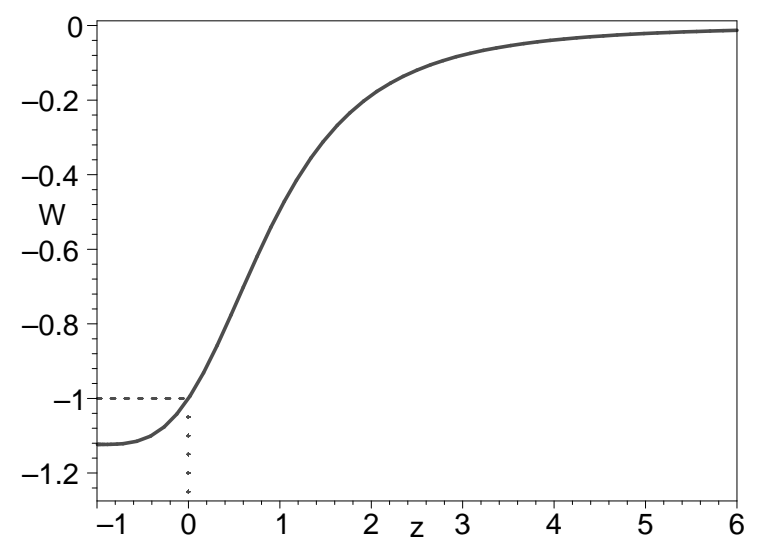

FIG. 1: Evolution of the equation of state $w$ for the Ricci dark energy as a function of redshift $z$.

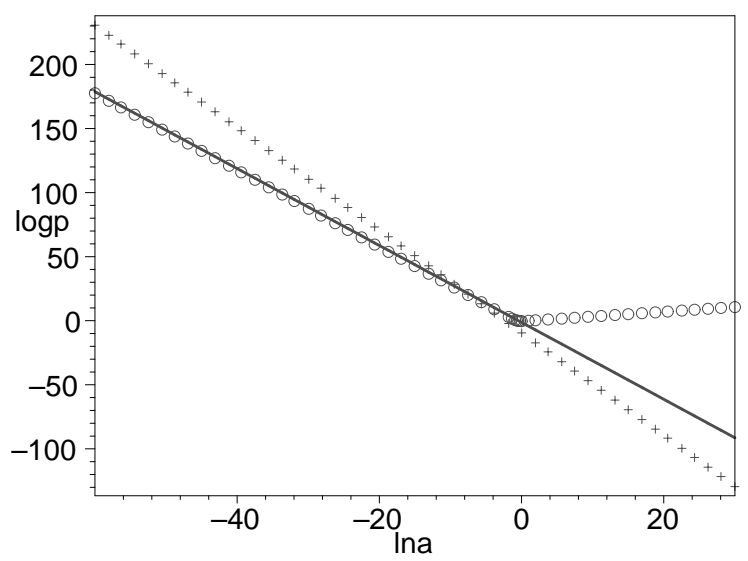

FIG. 2: Evolution of the radiation density (crosses), nonrelativistic matter density (solid line) and Ricci dark energy density (circles) $\log \rho$ as the function of $\ln a$.

At high redshifts, the equation of state is nearly zero, so the Ricci dark energy behaves just like dark matter, with $\rho_{X} / \rho_{m} \simeq 0.29$. The equation of state $w$ approaches -1 at $z \sim 0$. In the distant future, the equation of state approaches $w=-1.12$, The Universe evolves into the phantom dominated epoch [12]. For this model, the equation state crosses -1, so it may be classified as a "quintom" [13].

In Fig 2, we plot the evolution of densities, $\log \rho$, for radiation (crosses), non-relativistic matter (solid line) and dark energy (circles) with $\ln a$. Here we have neglected phase transitions, new degrees of freedoms and transitions from non-relativistic to relativistic particles at high temperature, etc, which would not make qualitative difference in the result. In this model the densities of nonrelativistic matter and dark energy were comparable with each other in the past Universe, but the acceleration be- gan at low redshift, so the coincidence problem is solved. The dark energy component made negligible contribution in the epoch of radiation dominated Universe, hence the standard Big-Bang Nucleosynthesis (BBN) model needs no revision.

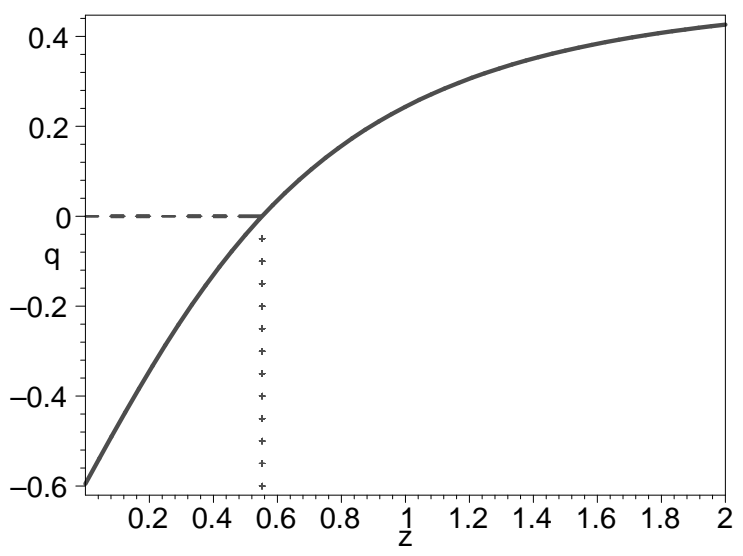

FIG. 3: Evolution of the deceleration parameter with redshift.

In Fig 3, we plotted the evolution of deceleration parameter,

$$
q \equiv \frac{1}{2}\left(1+\frac{3 p_{t o t}}{\rho_{t o t}}\right)=\frac{1}{2}+\frac{3 p_{X}}{2 \rho_{X}+2 \rho_{m}},
$$

where $p_{t o t}, \rho_{t o t}$ denote the total pressure and density of the Universe respectively. The acceleration of the Universe starts at $z \simeq 0.55$. For comparison, joint analysis of $\mathrm{SNe}+\mathrm{CMB}$ data with the $\Lambda \mathrm{CDM}$ model yields $z_{T}=0.52-0.73$ [14].

In Fig, we plotted the evolution of age of the Universe

$$
t=\frac{1}{H_{0}} \int_{0}^{\frac{1}{1+z}} \frac{d x}{h} .
$$

Three circles denote the ages of several old objects, LBDS 53W091 $(z=1.55, t=3.5 \mathrm{Gyr})$ [15], LBDS 53W069 $(z=1.43, t=4.0 \mathrm{Gyr})[16$ ] and APM $08279+5255$ $(z=3.91, t=2.1$ Gyr $)$ [17]. H. Wei and S. N. Zhang [18] recently pointed out that the ages of these three old high redshift objects are inconsistent with the holographic dark energy model of Ref. [8]. However, as shown in Fig.4, our model of Ricci dark energy does not suffer from this age problem.

\section{STRUCTURE FORMATION}

From Eq. (9), we see that if $\alpha \approx 0.46$ which yields the correct dark energy density and equation of state today, then the dark energy component behaves much as dust matter during most of the epoch of matter domination. Thus at earlier time the matter component is greater than in the best fit $\Lambda \mathrm{CDM}$ model, the difference could 


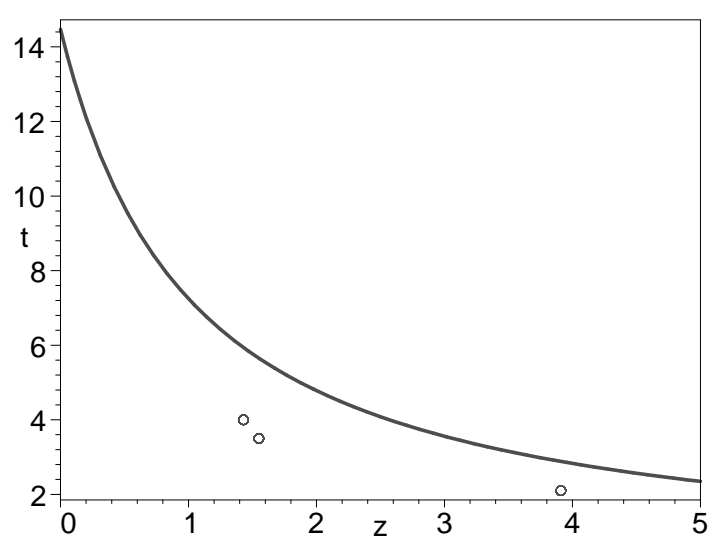

FIG. 4: Age of the Universe with redshift. Three circles denote the ages of three old objects, LBDS 53W091, LBDS 53W069 and APM 08279+5255.

be as large as about 30\%. An obvious question then is whether this would affect the growth rate of density perturbation, and upset the usual structure formation scenario?

We now investigate this problem with numerical calculations by using a modified version of the Boltzmann code $C A M B$ [19]. In Figure [5, we compare the evolution of density perturbations in our model with that in the $\Lambda \mathrm{CDM}$ model. The parameters as discussed earlier are $\Omega_{k 0}=0, \Omega_{m 0}=0.27\left(\Omega_{b}=0.04, \Omega_{c}=0.23\right), \Omega_{X 0}=0.73$, and $w_{0}=-1$.

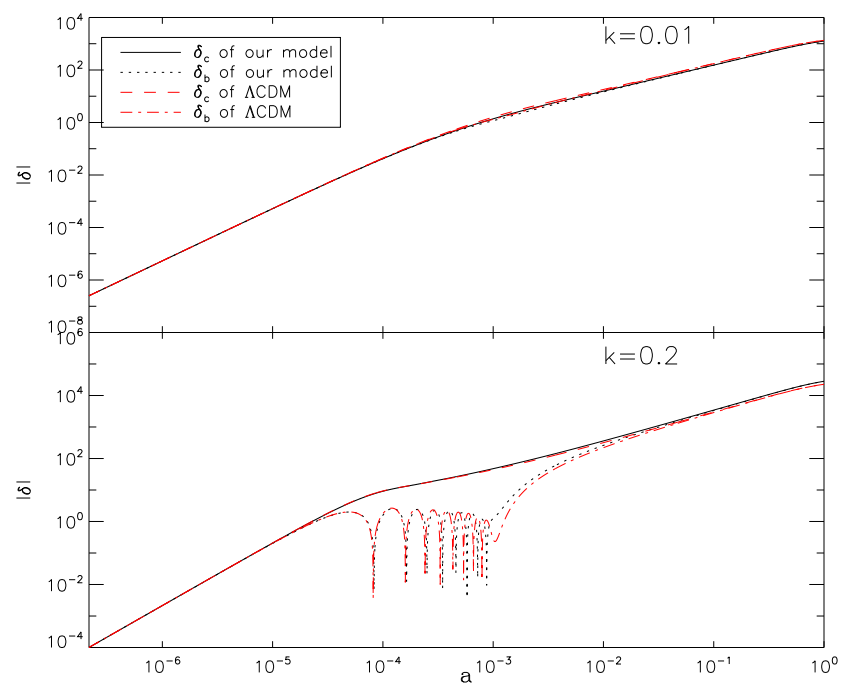

FIG. 5: Evolution of the density perturbations in the Ricci dark energy model and $\Lambda$ CDM model for two wavenumbers: $\mathrm{k}=0.01$ (upper panel) and $0.2 \mathrm{~h} \mathrm{Mpc}^{-1}$ (lower panel). $\delta_{c}$ and $\delta_{b}$ are the perturbations of cold dark matter and baryon respectively.

Figure 5 shows that for the large scale perturbations (e.g. $\mathrm{k}=0.01 \mathrm{~h} \mathrm{Mpc}^{-1}$ modes) the differences between our model and the $\Lambda \mathrm{CDM}$ model are very small, almost invisible. For the small scale perturbations (e.g. $\mathrm{k}=0.2 \mathrm{~h} \mathrm{Mpc}^{-1}$ modes), the amplitudes of $\delta_{c}$ and $\delta_{b}$ in our model are slightly (about 20\%) larger than these in $\Lambda \mathrm{CDM}$ model, due to the extra dust-like component in our model.

We plot the matter power spectra at different redshifts in Fig. 6. As expected, due to the extra dust-like component, the matter-radiation equality occurred at smaller $a_{e q}$, so the turn over in the matter power spectrum are also at smaller scales. Also, the growth rate of our model differs from that in $\Lambda \mathrm{CDM}$ model. However, the deviation of the shapes of the spectra from $\Lambda$ CDM model is not too large, it is expected that the observation could be fitted well by adjusting other parameters, such as $\sigma_{8}$ and $n_{S}$.

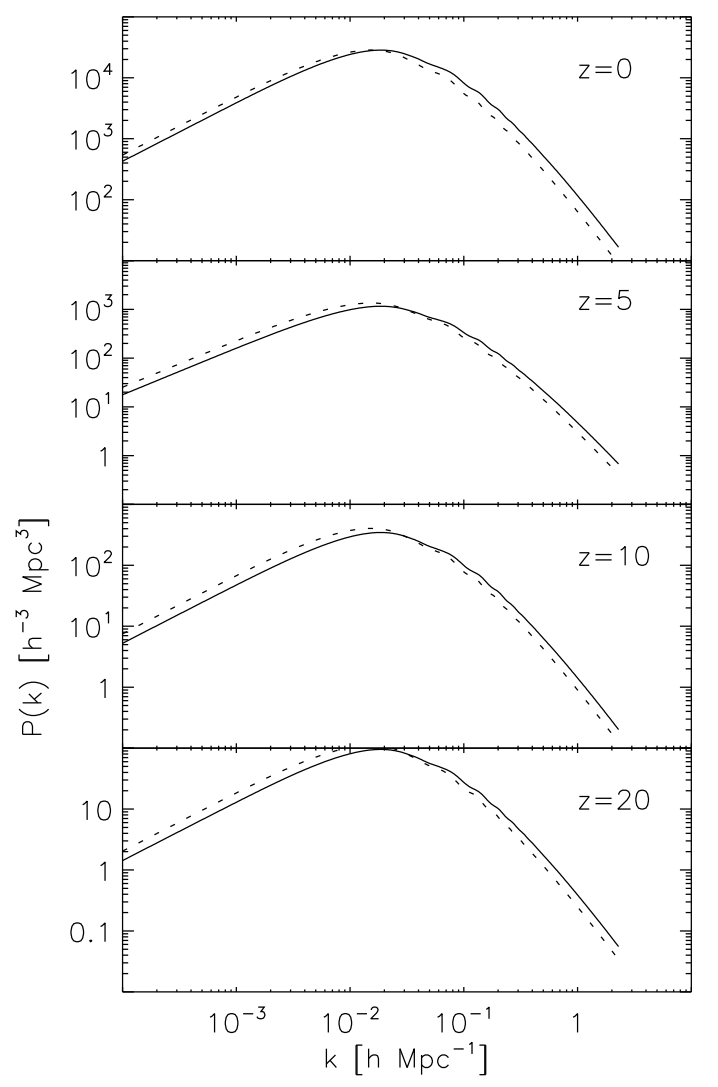

FIG. 6: The matter power spectra at different redshifts. From top to bottom: $\mathrm{z}=0, \mathrm{z}=5, \mathrm{z}=10$ and $\mathrm{z}=20$. The solid and dashed curves represent our model and $\Lambda$ CDM model respectively.

We have also calculated the angular spectrum of CMB temperature anisotropy, as shown in Fig.7. It differs from that of $\Lambda \mathrm{CDM}$ model mostly at small scales, where the experimental error bars are still very large, so our model is not in conflict with current CMB observations. Future high precision observations (e.g. from the Planck satellite) may help distinguish our model from the $\Lambda \mathrm{CDM}$ model. 


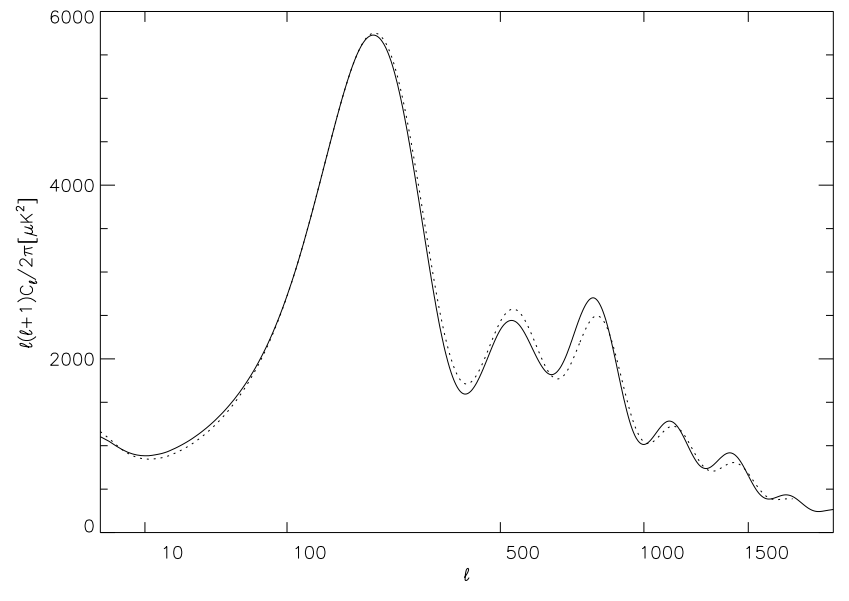

FIG. 7: The theoretical CMB TT spectrum of our model (solid curve) compared with $\Lambda$ CDM model (dashed curve). We normalize two amplitudes of spectra to same at the first peak.

At first sight, it may be a little surprising that such large extra contribution to the dust-like matter produce only such small difference. To better understand this, we plot the time evolution of the Hubble parameter in our model and the $\Lambda$ CDM model in Fig. 8. As can be seen from the figure, during the radiation dominated era, the Hubble rates are almost identical-for the matter contribution is dynamically negligible then. In the matter dominated era, there is some difference, but the largest difference is only $10 \%$ at $a=10^{-2}$. Then as dark energy dominates, again the difference between the two models become very small. We know that during the matter dominated epoch, the growth rate of density fluctuation is proportional to scale factor $a$, since the difference in expansion history is small, so the modification to structure formation history is also slight.

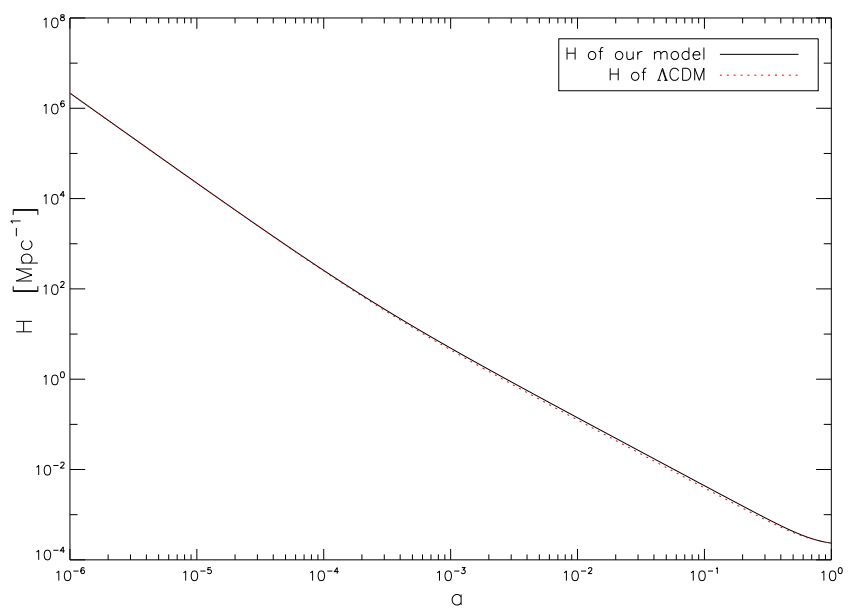

FIG. 8: Evolution of the Hubble parameter of our model and the $\Lambda$ CDM model.

\section{DISCUSSION}

We have shown that if we replace the future event horizon in the holographic dark energy model with the Ricci scalar curvature radius, i.e., $\rho_{X} \propto R$, then the resulting Ricci dark energy model is viable phenomenologically. The expansion history of the Universe in this model is similar to that of $\Lambda$ CDM at late times which has probed by supernovae observation. The model is also free of the age problem, which plagues the holographic dark energy model. We have only illustrated our model with one simple example. For different cosmological parameters, the model differs slightly. The best fit parameters and their allowed ranges will be investigated in future work.

We have also studied the structure formation in our model. The result shows that despite of the dust-like contribution from the dark energy component during the epoch of radiation domination, the matter power spectrum and CMB angular spectrum deviates from the $\Lambda \mathrm{CDM}$ case only slightly. In this paper we have only considered the large scale effect of $\rho_{X} \propto R$. In fact, due to inhomogeneities, $R$ has spatial fluctuations. The effect of such fluctuations will also be dealt with in our future study.

Our model has avoided the casuality problem of holographic dark energy, because the dark energy is determined by the locally determined Ricci scalar curvature, not the future event horizon. Moreover, in our model the fine tuning problem is avoided, because the dark energy is not associated Planck or other high energy physics scale, but with the size of space-time curvature. Interestingly, the coincidence problem is also solved in this model: as the dark energy is proportional to $R$, it is relatively small during radiation dominated $\mathrm{era}^{1}$. Once entering the matter dominated era, $R^{1 / 2}$ become comparable to $H$, the dark energy also become dynamically significant, and is always comparable to the size of the non-relativistic matter by construction. The change from deceleration to acceleration happens near $a \sim 1$ with very plausible model parameters.

While this model is phenomenologically viable and also solves the fine tuning problem and coincidence problem, one may question whether there is any physical mechanism or reasoning for which such a model could be motivated. As there is so much things unknown and uncertain in quantum gravity at present, it is difficult to make a very definite answer to this question. Rather, we take the view that the phenomenological success of our model may motivate theorists to look for such mechanism in their investigations of quantum gravity. However, we do try to answer this problem from the following two perspectives.

\footnotetext{
1 The actual value of the $R$ is not necessarily small during radiation domination, but its value relative to the Hubble rate is small so its influence on dynamics of expansion can be neglected. See Ref. 21] for discussion on this point.
} 
First, we considered the construction of a K-essence model 22] which reproduce the behavior of our model. The K-essence is a "designer fluid" with adjustable equation of state, which was first proposed as a model of dark energy to solve the coincidence problem. Moreover, the $\mathrm{K}$-essence equation of state can also cross -1 [23]. As such, it is not surprising that for each of our model with a given set of model parameters, one can build a model of K-essence to mimic its behavior. We describe the construction of such a model in the Appendix. Interestingly, for small $\alpha$ the $\mathrm{K}$-essence action reduces to the recently proposed Cuscuton action [24]. We find that the speed of sound in this model is positive, so it is classically stable. However, the fundamental dynamics of the K-essence model are of course not the same as that of our model, so the K-essence model can not realize the general mechanism of self-adjustment of our model.

Second, we may also consider some heuristic arguments based on conjectures about quantum gravity effects, though of course the argument is unavoidably vague and speculative before we have a better understanding of quantum gravity. An an example, one may suppose that the dark energy raises due to fluctuation in quantum gravity. The gravitational action is given by

$$
S=\frac{1}{16 \pi} \int d^{4} x \sqrt{-g} R .
$$

In classical evolution, $\delta S=0$. However, in quantum gravity, at some minimal scale, it has been suggested 25] that this action might be small but non-zero due to quantum fluctuation, $\Delta S \simeq 1$ within the space-time volume $\Delta V \Delta t$, so that

$$
\Delta S=\frac{R}{16 \pi} \Delta V \Delta t \sim 1 .
$$

The density fluctuation in such small region is given by the energy-time uncertainty relation, $\Delta E \Delta t \sim 1$, so

$$
\rho_{X} \sim \frac{\Delta E}{\Delta V} \propto R
$$

which is what we have taken as the hypothesis of our model.

Finally, we point out that although we have been motivated by the holographic principle when constructing our model, the Ricci dark energy does not necessarily have to be connected with the holographic principle. The dark energy associated with the Ricci scalar may also arise for other physical reasons. The main point of this paper is to show that dark energy of such property would be successful.

\section{APPENDIX A: K-ESSENCE CONSTRUCTION}

Here we show that for each of our model, one can also construct a K-essence model [22] which mimics its behavior.
As shown in the section II, the equation of state of the Ricci scalar dark energy may cross over -1 . This can be realized with $\mathrm{K}$-essence field with Lagrangian density $p(\phi, X)$ [23] where $\phi$ a scalar field.

Now let us consider the Lagrangian density of a Kessence field $p(\phi, Y)$, where $Y=-\frac{1}{2} \partial_{\mu} \phi \partial^{\mu} \phi$ is the kinetic energy term. We note that since we chose the signature of the metric as $(-1,+1,+1,+1),$,$Y is positive.$ For simplification, here we consider the pure K-essence with the Lagrangian density

$$
p=p(Y) .
$$

Identifying the energy momentum tensor of the scalar field with that of a perfect fluid, we can easily derive the energy density and pressure:

$$
\rho_{Y}=2 Y \frac{d p}{d Y}-p, \quad p_{Y}=p
$$

Put $\rho_{X}=\rho_{Y}$ and $p_{X}=p_{Y}$, we have

$$
\begin{aligned}
2 Y \frac{d p}{d Y}-p & =\frac{\alpha}{2-\alpha} \Omega_{m 0} e^{-3 x}+f_{0} e^{-\left(4-\frac{2}{\alpha}\right) x}, \\
p & =-\left(\frac{2}{3 \alpha}-\frac{1}{3}\right) f_{0} e^{-\left(4-\frac{2}{\alpha}\right) x} .
\end{aligned}
$$

Solving Eq.(A3), we find a remarkably simple form for the action of the pure K-essence field

$$
\begin{aligned}
S_{K} & =\frac{1}{16 \pi} \int d^{4} x \sqrt{-g} p \\
& \propto \frac{1}{16 \pi} \int d^{4} x \sqrt{-g}\left(\sqrt{-\frac{1}{2} \partial_{\mu} \phi \partial^{\mu} \phi}-V_{0}\right)^{\frac{2(1-2 \alpha)}{2-\alpha}}
\end{aligned}
$$

where $V_{0}$ is a positive integration constant. We note that when $\alpha \ll 1$, this action reduces to the Cuscuton action with a constant scalar potential which were proposed by N. Afsgordi, D. J. H. Chung and G. Geshnizjani recently 24]. The energy density of this model is easily shown to be positive using Eq. A2 . The solution is stable with respect to small perturbation to the Cauchy data if the effective sound speed is positive. The sound speed of $\mathrm{K}$-essence is given by [22]

$$
v_{s}^{2} \equiv \frac{p_{Y, Y}}{\rho_{Y, Y}}=\frac{\left(\sqrt{Y}-V_{0}\right)(\alpha-2)}{3 \alpha \sqrt{Y}} .
$$

Since $\alpha \simeq 0.46$, so if $\sqrt{Y} \leq V_{0}$, we will have $v_{s} \geq 0$. Thus we have a viable K-essence model which mimics the behavior of our model.

\section{ACKNOWLEDGMENTS}

We thank professors Miao Li, Ronggen Cai, Chongming Xu, Pengjie Zhang and Andrew R. Liddle for helpful discussions. This work is supported by the National Science Foundation of China under the Distinguished Young Scholar Grant 10525314, the Key Project 
Grant 10533010, and Grant 10575004; by the Chinese Academy of Sciences under grant KJCX3-SYW-N2; and by the Ministry of Science and Technology under the National Basic Sciences Program (973) under grant 2007 CB815401.
[1] S. Perlmutter et al., Astrophys. J. 517, 565 (1999)

[2] A. G. Riess et al., Astron. J. 116, 1009 (1998)

[3] S. Weinberg, astro-ph/0005265

[4] see e.g. G. t' Hooft, gr-qc/9310026 hep-th/0003004 L. Susskind, J. Math. Phys. 36, 6377 (1995) (hep-th/9409089); J. M. Maldacena, Adv. Theor. Math. Phys. 2, 231 (1998); W. Fischler and L. Susskind, hep-th/9806039 R. Bousso, Rev. Mod. Phys. 74, 825 (2000).

[5] A. Cohen, D. Kaplan and A. Nelson, Phys. Rev. Lett. 82 (1999) 4971; P. Horava and D. Minic, hep-th/hepth/0001145, Phys.Rev.Lett. 85 (2000) 1610; S. Thomas, Phys. Rev. Lett. 89 (2002) 081301.

[6] W. Fischler and L. Susskind, hep-th/9806039 R. Bousso, JHEP 9907 (1999) 004.

[7] S. D. H. Hsu, hep-th/0403052

[8] M. Li, Phys.Lett. B603 (2004) 1.

[9] R. Horvat, Phys. Rev. D70 (2004) 0873001; D. Pavon and W. Zimdahl, Phys. Lett. B628 (2005) 206; B. Guberina, R. Horvat and H. Nikolic, JCAP 0701 (2007) 012; X. Zhang, F. Q. Wu, Phys. Rev. D 72 (2005) 043524; Z. Chang, F.Q. Wu, X. Zhang, Phys.Lett.B 633 (2006) 14; X. Zhang, F. Q. Wu, Phys.Rev.D76 (2007) 023502; X. Zhang, Int.J.Mod.Phys.D 14 (2005) 1597; M. R. Setare, J. Zhang, X. Zhang, JCAP 0703 (2007) 007; J. Zhang, X. Zhang, H. Liu, Phys.Lett.B659 (2008) 26; X. Zhang, Phys.Lett.B 648 (2007) 1; X. Zhang Phys. Rev. D74 (2006) 103505,2006; J. Zhang, X. Zhang, H. Liu Phys.Lett.B651 (2007) 84; Y. Ma, X. Zhang, Phys.Lett.B 661 (2008) 239; J. Zhang, X. Zhang, H. Liu, Eur.Phys.J.C52 (2007) 693; L. Xu, W. Li J. Lu arXiv: 0810.4730 (to appear in MPLA).

[10] R. G. Cai, arXiv:0707.4049v3; I. P. Neupane, Phys. Rev. D 76, 123006 (2007); I. P. Neupane, arXiv: 0708.2910.

[11] D. N. Spergel et al., Astrophys. J. Suppl. Ser. 170,
377 (2007); Morad Amarzguioui, O. Elgaroy, David F. Mota and T. Multamaki, Astrophys. J. 454, 707 (2006).

[12] R. R. Caldwell, Phys Lett. B 545 (2002) 23.

[13] B. Feng, X. L. Wang and X. M. Zhang, Phys. Lett. B 607, 35 (2005) astro-ph/0404224; B. Feng, M. Li, Y. S. Piao and X. M. Zhang, Phys. Lett. B 634, 101 (2006) astro-ph/0407432;

[14] U. Alam, V. Sahni and A. A. Starobinsky, JCAP 0406, 008 (2004); Z. H. Zhu, M. K. Fujimoto and X. T. He, Astrophys. J. 603, 365 (2004)

[15] J. Dunlop et al., Nature 381, 581 (1996).

[16] H. Spinrad et al., Astrophys. J. 484, 581 (1999).

[17] D. Jain and A. Dev, Phys. Lett. B 633, 436 (2006) astro-ph/0509212.

[18] H. Wei and S. N. Zhang, Phys. Rev. D 76, 063003 (2007)

[19] A. Lewis and A. Challinor, http://camb.info/.

[20] E. Komatsu et al. [WMAP Collaboration], arXiv:0803.0547 [astro-ph].

[21] X. Chen and M. Kamionkowski, Phys. Rev. D 60 (1999) 104036.

[22] C. Armendariz-Picon, V. F. Mukhanov and P. J. Steinhardt, Phys. Rev. Lett. 85, 4438 (2000) astro-ph/0004134; C. Armendariz-Picon, V. F. Mukhanov and P. J. Steinhardt, Phys. Rev. D 63, 103510 (2001) astro-ph/0006373;

[23] A. Vikman, Phys. Rev. D 71, 023515 (2005) astro-ph/0407107]; S. Tsujikawa, Phys. Rev. D 72, 083512 (2005) astro-ph/0508542; E. J. Copeland, M. Sami and S. Tsujikawa, hep-th/0603057.

[24] N. Afshordi, D. J. H. Chung, and G. Geshnizjani, Phys. Rev. D75 083513 (2007), hep-th/0609150 G. Robbers, N. Afshordi, M. Doran, Phys. Rev. Lett. 100 (2008) 111101.

[25] L. J. Garay, Int. J. Mod. Phys. A10 (1995) 145-166. 